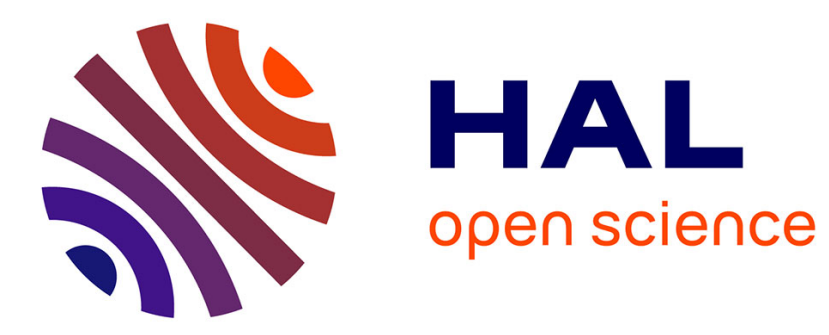

\title{
Caractérisation électrique de films amorphes de As50Te 50 irradiés par des ions lourds
}

\author{
M. Benmalek, J.M. Mackowski, J. Tousset
}

\section{To cite this version:}

M. Benmalek, J.M. Mackowski, J. Tousset. Caractérisation électrique de films amorphes de As50Te 50 irradiés par des ions lourds. Revue de Physique Appliquée, 1977, 12 (5), pp.707-710. 10.1051/rphysap:01977001205070700 . jpa-00244230

\section{HAL Id: jpa-00244230 https://hal.science/jpa-00244230}

Submitted on 1 Jan 1977

HAL is a multi-disciplinary open access archive for the deposit and dissemination of scientific research documents, whether they are published or not. The documents may come from teaching and research institutions in France or abroad, or from public or private research centers.
L'archive ouverte pluridisciplinaire HAL, est destinée au dépôt et à la diffusion de documents scientifiques de niveau recherche, publiés ou non, émanant des établissements d'enseignement et de recherche français ou étrangers, des laboratoires publics ou privés. 


\title{
CARACTÉRISATION ÉLECTRIQUE DE FILMS AMORPHES DE $\mathrm{As}_{50} \mathrm{Te}_{50}$ IRRADIÉS PAR DES IONS LOURDS
}

\author{
M. BENMALEK, J. M. MACKOWSKI et J. TOUSSET \\ Institut de Physique Nucléaire (et IN2P3), Université Claude-Bernard, Lyon I, \\ 43, Boulevard du 11 Novembre 1918, 69621 Villeurbanne, France
}

\begin{abstract}
Résumé. - Des films amorphes de composition $\mathrm{As}_{50} \mathrm{Te}_{50}$ sont irradiés avec des ions azote et néon d'énergie comprise entre 0,5 et $2 \mathrm{MeV}$. L'évolution de leur résistance électrique est suivie pendant et après l'irradiation. La diminution de résistivité est corrélée à l'état initial de la couche ; en particulier, le recuit initial-prérecuit module l'amplitude de cette décroissance.

L'accroissement de conductivité est attribué à une augmentation du nombre des défauts structuraux, lesquels provoquent une modification de la densité des états localisés dans la bande interdite.

Abstract. - Amorphous As ${ }_{50} \mathrm{Te}_{50}$ films have been irradiated with Nitrogen and Neon ions in the energy range between 0.5 to $2 \mathrm{MeV}$. The electrical resistance has been monitored during the irradiation and was found to decrease. The magnitude of this decrease was depending on whether the films were preannealed or not. The conductivity enhancement is interpreted in terms of structural defects increases which give rise to density of localized states in the gap.
\end{abstract}

1. Introduction. - Les semiconducteurs amorphes possèdent une structure désordonnée qui leur confère des propriétés électriques peu sensibles aux radiations. Cette caractéristique est bien vérifiée pour des fluences neutroniques de $10^{18} \mathrm{n} / \mathrm{cm}^{2}$ et des doses gamma de $10^{8}$ rads [1]. L'obtention de doses supérieures est difficile à réaliser. De plus, la radioactivité induite lors de telles études constitue une complication supplémentaire. L'utilisation des ions énergétiques, tout en éliminant les difficultés citées, permet de simuler les fortes fluences par l'introduction de taux élevés de dommages. Dans le cas des semiconducteurs amorphes cette facilité a peu été utilisée jusqu'à maintenant [2].

Nous avons étudié les effets du bombardement ionique sur les propriétés électriques du binaire amorphe $\mathrm{As}_{50} \mathrm{Te}_{50}$ irradié par des ions Azote et Néon d'énergie comprise entre 0,5 et $2 \mathrm{MeV}$ et à des doses variant de $10^{11}$ à $10^{15}$ ions $/ \mathrm{cm}^{2}$.

2. Partie expérimentale. - Des couches amorphes de 1 à $2 \mu \mathrm{m}$ de AsTe sont déposées par pulvérisation radiofréquence. Les épaisseurs sont déterminées par la méthode Nomarsky. Trois électrodes en or sont évaporées thermiquement suivant une géométrie coplanaire telle que l'irradiation puisse s'effectuer sur un seul des gaps ainsi définis.

Les ions $\mathrm{N}^{+}$et $\mathrm{Ne}^{+}$utilisés sont délivrés par l'accélérateur Van de Graaff de l'Institut de Physique Nucléaire de Lyon. Cette machine produit un faisceau de quelques milliampères et il a été nécessaire de le réduire pour travailler à faible dose.

La figure 1 décrit le système d'irradiation. Sur la trajectoire du faisceau nous avons intercalé deux dia-

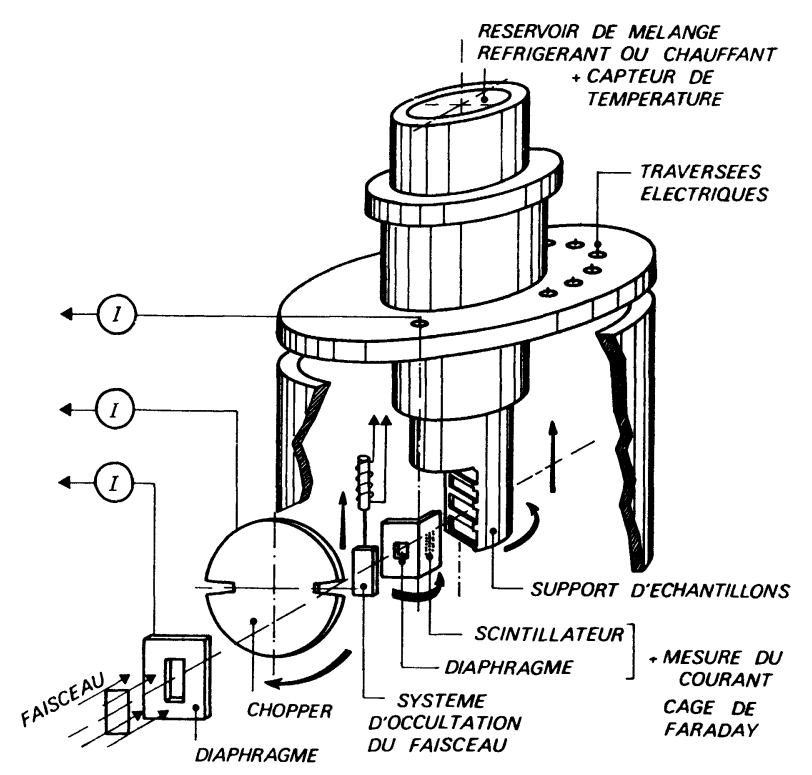

FIG. 1. - Schéma du dispositif d'irradiation.

phragmes et un chopper pour que la lame ne reçoive qu'une fraction du courant initial. Une cage de Faraday escamotable en permet la mesure. Avec un tel système nous pouvons obtenir des taux de dose inférieurs à $10^{11}$ ions $/ \mathrm{cm}^{2} / \mathrm{s}$. La mesure de la résistance est effectuée à intervalles réguliers, après masquage de la lame au moyen d'un obturateur automatique. Ce dispositif permet d'une part de travailler dans le domaine des faibles doses $10^{11}-10^{12}$ ions $/ \mathrm{cm}^{2}$ et d'autre part de minimiser la composante thermique induite par le faisc eau d'ions dans la couche mince. 
3. Résultats et discussion. - Nous avons représenté, figure 2 , la variation relative de la résistance $R / R_{0}$ en fonction du temps. Sous irradiation on observe une décroissance de la résistance (courbe $\mathrm{A}$ ) puis à la fin de

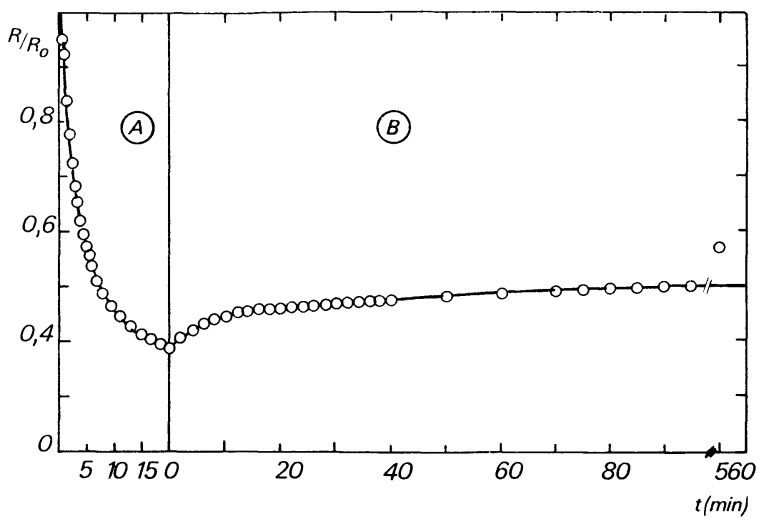

Fig. 2. - Variation de la résistance d'un film de AsTe pendant l'irradiation avec des ions $\mathrm{N}^{+}$de $1 \mathrm{MeV}(\mathrm{A})$ et après l'irradiation (B).

celle-ci une récupération (courbe B), qui se traduit par une remontée de la résistance; cette dernière peut d'ailleurs retrouver sa valeur initiale si la dose reçue est faible. Ce type de comportement a déjà été observé pour d'autres semiconducteurs amorphes tels que le germanium, le silicium ou les systèmes AsTeS et AsTeSe [3, 4, 5].

La variation de la résistance en fonction de la dose peut être décrite selon 3 zones comme on peut le voir sur la figure 3 qui représente les variations $\Delta R / R_{0}$

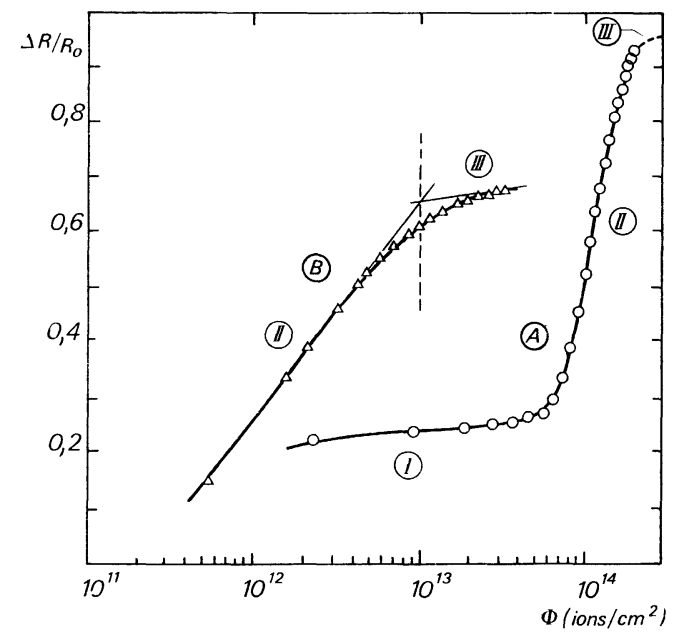

FIg. 3. - Influence du prérecuit sur la variation $R_{0}-R / R_{0}$ de la résistance pendant l'irradiation $\left(\mathrm{N}^{+}, 0,5 \mathrm{MeV}\right): \mathrm{A}:$ Film prérecuit $30 \mathrm{~min}$. à $121^{\circ} \mathrm{C}$; $\mathrm{B}$ : Film non prérecuit.

pour deux films de AsTe. Pour un film prérecuit (courbe $\mathrm{A}$ ) on observe une première zone correspondant à une variation lente de la résistance suivie d'une deuxième zone de variation rapide. La troisième zone correspond à une saturation ; celle-ci est peu visible sur cette courbe. Pour le film n'ayant pas subi de prérecuit (courbe B), le premier palier est absent et l'on a seulement les zones II et III ; de plus la courbe est décalée de plus d'une décade par rapport à celle du film prérecuit. On sait que le prérecuit guérit les défauts formés lors du dépôt de la couche mince ; il se traduit dans ce cas par l'augmentation de la dynamique tant en dose déposée qu'en amplitude de variation de la résistance.

Afin de mettre en évidence la loi de variation de la conductivité pour la zone II, nous avons tracé le complément de $\Delta R / R_{0}$ soit $R / R_{0}$ en fonction de la dose suivant une échelle semi-logarithmique. La figure 4 montre une variation linéaire qui indique clairement la loi exponentielle de variation de la conductivité pour cette zone II.

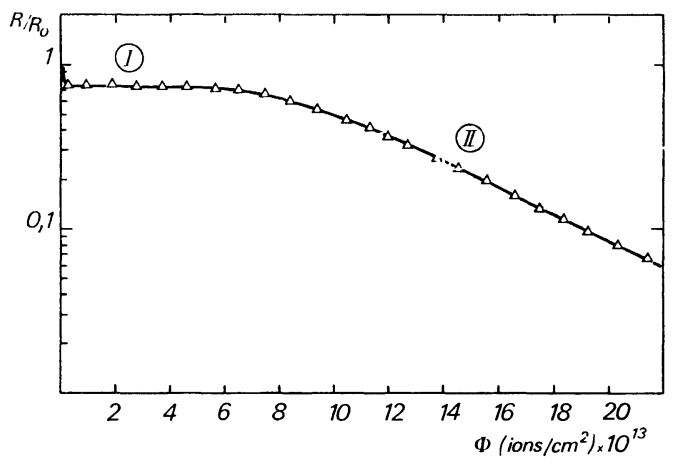

FIG. 4. - Représentation semi-logarithmique de la variation de la résistance en fonction de la dose pour le film prérecuit. La partie linéaire décroissante correspond à la zone II de la figure 3 .

Pour mieux illustrer la variation de la résistance des films amorphes sous irradiation nous l'avons comparée à celle d'un film mince cristallisé après dépôt. La cristallisation du film a été vérifiée d'une part par la valeur $\mathrm{du}$ rapport des résistivités $\rho_{\text {cristal }} / \rho_{\text {amorphe }}=10^{-6}$ et d'autre part par analyse thermique différentielle. Sur la figure 5 la courbe 1 est caractéristique d'un film prérecuit avec les étapes I et II, la courbe 2 correspond au film cristallisé. On voit que le rapport $R / R_{0}$ croît pour le film polycristallisé, comme cela a déjà été observé par certains auteurs pour des films cristallisés d'oxydes ou de métaux [6]. On attribue généralement cette augmentation de la résistance à la désorganisation $\mathrm{du}$ réseau périodique.

La figure 6 montre les variations de la résistance pour deux séries de films irradiés à différentes températures.

Les films $1 a$ et $2 a$ ont été irradiés à la température ambiante sans prérecuit et servent de témoins. Le film $1 b$ a été maintenu pendant 1 heure à $110^{\circ} \mathrm{C}$ puis irradié à cette température. Le film $2 b$ a subi un prérecuit de 2 heures 30 à $90^{\circ} \mathrm{C}$ puis a été irradié à cette température. On observe pour les 2 films irradiés à haute température l'absence de saturation à la dose atteinte, alors que pour les films témoins ( $1 a$ et $2 a$ ) celle-ci est bien marquée. D'autre part le film irradié à $110^{\circ} \mathrm{C}$ 


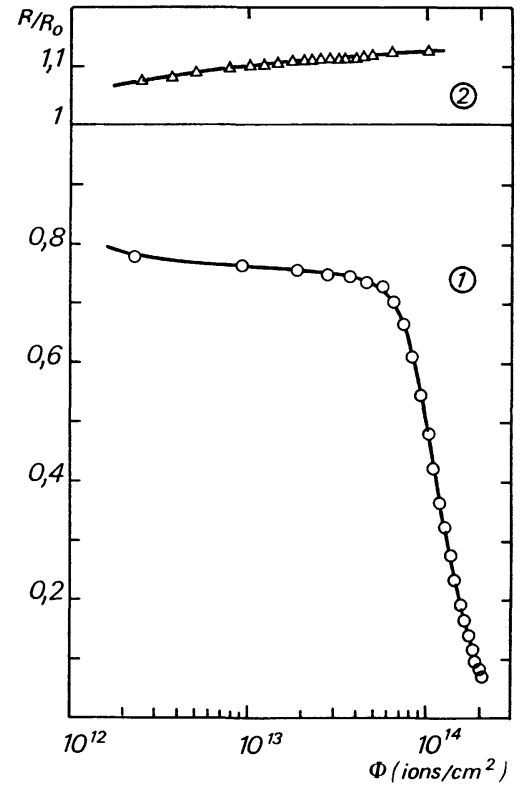

Fig. 5. - Comparaison des comportements de deux films de AsTe sous irradiation $\left(\mathrm{N}^{+}, 0,5 \mathrm{MeV}\right)$. (1) Film amorphe prérecuit ; (2) Film cristallisé.

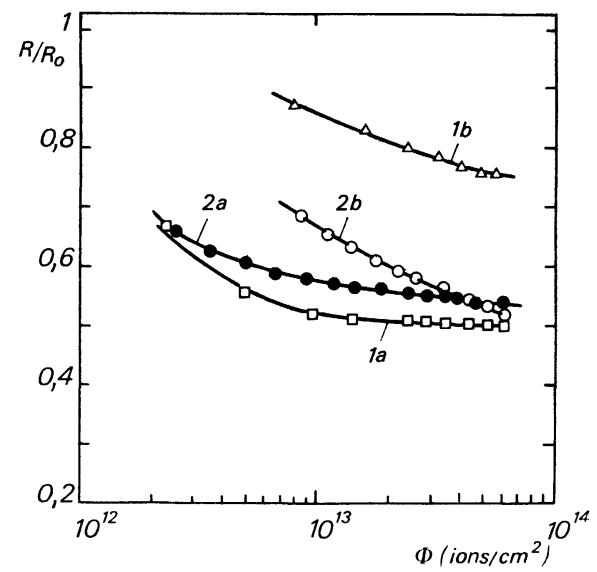

FIG. 6. - Variation de la résistance pour 2 séries de films irradiés à des températures différentes $\left.\left(\mathrm{N}^{+}, 1 \mathrm{MeV}\right), 1 a, 2 a\right):$ Films non prérecuits irradiés à l'ambiante; $1 b)$ Film prérecuit $1 \mathrm{~h}$ et irradié à $\left.100^{\circ} \mathrm{C} ; 2 b\right):$ Film prérecuit $2 \mathrm{~h} 30$ et irradié à $90^{\circ} \mathrm{C}$. :

présente une variation de résistance plus faible, ce qui indique que le processus de guérison des défauts est activé thermiquement.

Les courbes d'Arrhenius pour un film irradié et son témoin sont représentées sur la figure 7. La courbe du film témoin est caractéristique d'une conductivité intrinsèque. Pour le film irradié on observe une baisse de l'énergie d'activation qui indique l'apparition de niveaux de « Hopping ». Ceci a d'ailleurs été observé sur des composés tels que $\mathrm{Ge}, \mathrm{As}_{2} \mathrm{Se}_{3}, \mathrm{PbI}_{2}$, irradiés, et certains auteurs l'attribuent à un déplacement du niveau de Fermi vers le bord de mobilité $[3,5]$. Ce déplacement se traduit aussi par une translation du

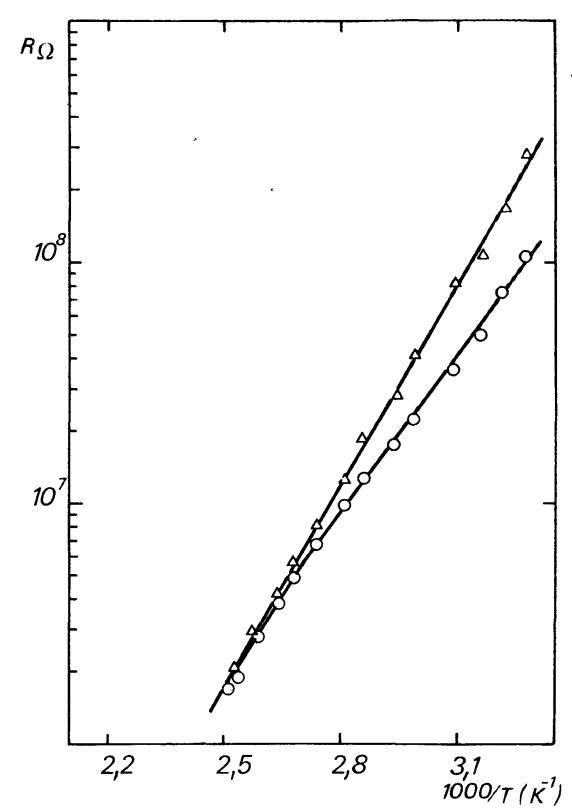

Fig. 7. - Courbes d'Arrhenius de deux films de AsTe : Irradié $\left(\mathrm{N}^{+}, 1 \mathrm{MeV}, 10^{15}\right.$ ions $\left./ \mathrm{cm}^{2}\right) ; \triangle$ : Témoin.

front d'absorption optique vers les faibles énergies comme on peut le voir sur la figure 8 représentant les courbes de coefficients d'absorption optique $\alpha$ en fonction de l'énergie des photons absorbés. Par compa-

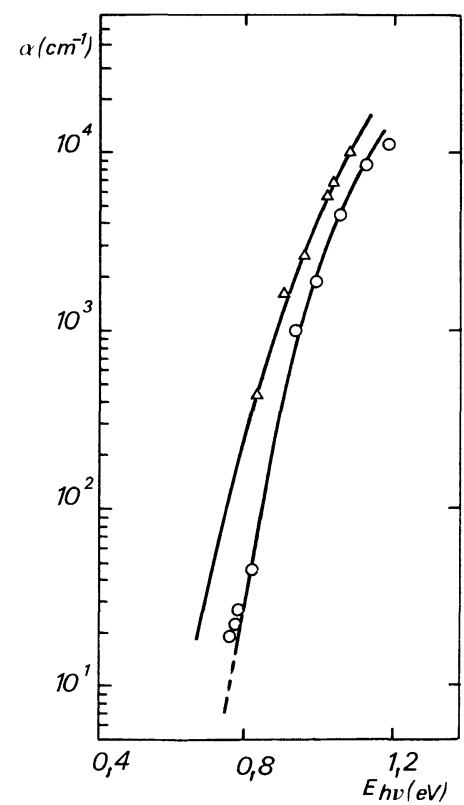

FIG. 8. - Courbes du coefficient d'absorption optique pour 2 films de AsTe. O Irradié $\left(\mathrm{N}^{+}, 0,5 \mathrm{MeV}, 10^{15}\right.$ ions $\left./ \mathrm{cm}^{2}\right)$. $\triangle$ Témoin.

raison, l'effet de prérecuit déplace la courbe $\alpha=f(h v)$ vers les hautes énergies [7]. D'après Olley [8], l'existence d'un tel phénomène aussi bien dans les corps cristallins [9] que dans les amorphes est plutôt lié à 
l'élargissement de l'absorption excitonique par irradiation.

Les résultats obtenus (avec les ions Azote) ne sont pas liés à la nature chimique de l'ion incident. En effet, des observations analogues ont été faites après irradiation avec des ions néon.

La microscopie électronique à balayage n'a révélé aucune modification de l'état des échantillons irradiés. L'évolution de nos couches est donc essentiellement liée aux modifications structurales induites par l'irradiation.

Les effets induits par bombardement ionique sont liés aux phénomènes de ralentissement de l'ion dans le corps solide. Un ion pénétrant dans une cible perd son énergie suivant deux processus : l'un électronique, interaction avec le cortège, provoquant excitations et ionisations, l'autre atomique, interaction avec un atome, se traduisant par des déplacements d'atomes qui modifient l'ordre initial.

La perte d'énergie totale est la somme de celles dues aux deux processus. Le rapport des pertes d'énergie dépend, pour un couple ion-cible donné, de l'énergie initiale de l'ion. A faible énergie $(\sim \mathrm{keV})$ la perte d'énergie atomique (ou nucléaire) domine, alors qu'en général au-dessus du $\mathrm{MeV}$ la perte d'énergie électronique devient plus importante. Dans notre cas la perte d'énergie électronique excède la perte d'énergie atomique d'un facteur 2 à $0,5 \mathrm{MeV}$ et d'un facteur 3 à $1 \mathrm{MeV}$. Cependant, les variations observées au niveau de la conductivité ne peuvent être mises sur le compte du seul processus électronique. En effet, des modifications similaires ont déjà été observées à plus faibles énergies [3, 10].

Donc, lors de l'irradiation les atomes sont soumis à des déplacements qui se traduisent par des liaisons cassées. Les défauts créés vont contribuer à la croissance de la conductivité. D'autre part les excitations électroniques vont influencer la formation d'excitons et les recombinaisons électron-trou modifiant aussi la conductivité. Après irradiation les centres non recombinés dont le nombre est plus élevé vont donner lieu à une conduction supplémentaire par saut. Si la dose est faible, par recombinaison sous l'effet de la température, ce nombre va très vite diminuer et l'on revient à la situation initiale. Par contre, à forte dose, le nombre de défauts structuraux induits est plus important et il est nécessaire de recourir à un recuit thermique pour obtenir la saturation des liaisons insatisfaites laissées par l'irradiation.
Après irradiation, la conductivité électrique à température ambiante peut s'écrire :

$$
\sigma=\sigma_{\mathrm{i}}+\sigma_{\mathrm{h}}
$$

avec $\sigma_{i}$ la conduction intrinsèque et $\sigma_{\mathrm{h}}$ la contribution prédominante due à l'irradiation.

Le mécanisme de modification de la conductivité par saut peut s'interpréter suivant deux modèles. Le premier implique que les défauts introduits par l'irradiation se traduisent essentiellement par l'augmentation de liaisons insatisfaites, ce qui donne lieu à une conduction par saut près du niveau de Fermi. Ce type de conduction décrit par la loi de Mott [11] est peu fréquent dans les chalcogénures et se manifeste surtout à basse température [10]. L'autre possibilité est la conduction liée aux queues de bandes. Ainsi l'irradiation en augmentant le désordre à grande distance contribue à l'accroissement de la densité des états localisés près des bords de bandes. Une telle modification peut conduire au déplacement du niveau de Fermi. Ceci est en bon accord avec les résultats des figures 7 et 8. On sait que dans les verres chalcogénures la présence de lone pair donne lieu à la formation d'états localisés près des bords de bandes ; nous pensons que, dans AsTe, les effets de l'irradiation doivent se manifester principalement à ce niveau.

Le recuit thermique, après irradiation, sature les liaisons insatisfaites, ce qui diminue la conduction par saut et l'on retourne à une conduction intrinsèque, observée expérimentalement.

4. Conclusion. - Ce travail a permis de montrer l'effet de l'irradiation par des ions énergétiques sur des semiconducteurs amorphes. Cet effet, bien que sensible au niveau des propriétés électriques, reste cependant assez faible à température ambiante en comparaison des modifications apportées dans les composés cristallins.

L'irradiation introduit des changements structuraux au niveau de l'ordre à courte distance sans transformation de l'état métastable. Ces changements structuraux se traduisent par l'augmentation de la conductivité dont la variation est expliquée en terme de conduction par saut. Cependant la compréhension globale de tous les phénomènes induits par l'irradiation nécessite des études dans le domaine des basses températures avec des couches dont les degrés de stabilisation sont parfaitement contrôlés.

\section{Bibliographie}

[1] HeNCH, L. L., J. Non-Crystal. Sol. 2 (1970) 250.

[2] Olley, J. A., Yoffe, A. D., Amorphous and liquid semiconductors (ed. Stuke J. et Brenig W. Taylor and Francis Ltd, Londres) 1974.

[3] Beyer, W., Stuke, J. and Wagner, H., Phys. Stat. Sol. (a), 30 (1975) 231.

[4] Olley, J. A., Solid State Commun. 13 (1973) 1441.

[5] Minami, T., Yoshida, A., TanaKa, M., J. Non-Crystal. Sol. 7 (1972) 326.
[6] Merkie, K. L., Singer, L. R., Appl. Phys. Lett. 11 (1967) 35.

[7] THEYE, M.L., Rapport DGRST, 73, 71070 (1975).

[8] Olley, J. A., Solid State Commun. 13 (1973) 1437.

[9] Meek, R. L., Gibson, W. M., McLennan, C. G., Maher, D. M., Appl. Phys. Lett. 20 (1972) 400.

[10] Benmalek, M., Thomas, J. P., Mackowski, J. M., Comm. à « Intern. Conf. on Ion Implantation in Semiconductors and Other Materials ", Boulder (USA) (1976).

[11] Motт, N. F., Phil. Mag. 19 (1969) 835. 